\title{
Effects of Sex Hormones on Food Intake, Body Weight, and Fat Composition: A Cross-Species Analysis
}

\author{
Sahara L. Ali ${ }^{1}$ and Megan G. Massa ${ }^{1 \#}$ \\ ${ }^{1}$ University of California, Los Angeles, CA, USA \\ \#Advisor
}

ABSTRACT

Reproductive hormones affect the physiology of eating, body weight, and fat composition differently among the sexes and across multiple species. While the reproductive influences on feeding are known, these studies have been previously limited to just one species in both studies and literature reviews. In addition, discrepancies have also been found across different species. For instance, female mice tend to experience no changes in food consumption whether estradiol is present or not, while female rats experience a decrease in food consumption with the presence of estradiol. The lack of cross-species comparison in these findings leads to a limited understanding of the overall effects of feeding and body composition. Not only are studies limited to one species, but studies are also limited to one sex. Not comparing results to the opposite sex prevents the consideration and realization of the discrepancies in the effects of hormones among the sexes. For example, men with higher levels of testosterone were correlated with healthy Body Mass Index (BMI) levels while women with higher levels of testosterone tend to weigh more than women with normal levels of testosterone. This literature review focuses on inter-species and sex differences of the effects of reproductive hormones on feeding, body weight, and fat composition.

\section{Introduction}

The physiology of eating is still not understood despite the increasing rate of obesity in western countries. However, several studies have shown that there may be an association between sex hormones, food intake, and body weight. In this literature review, we will first provide an overview of the general function of the different hormones that are in the body. Next, we investigate how the function of the presence or absence of estradiol, progesterone, and testosterone on food intake and body weight across several species. Next, we show how progesterone can interact with estradiol to affect these same parameters. Finally, we note how hormones can affect adipose tissue composition in several species.

\section{Introduction to Gonadal Hormones}

Hormones are known to regulate bodily functions and tend to target specific organs or tissues (Nelson, 2011). The hormone progesterone is known to regulate pregnancies and mating behavior in mammals (Nelson, 2011). Testosterone is an androgen that is important for spermatogenesis, maintenance of the genital tract, and maintenance of the accessory sex organs. (Nelson, 2011). Additionally, androgens are precursors of estrogens, and enzymes that are mainly present in the ovaries and brain convert testosterone and androstenedione into estrogens, estradiol and estrone, respectively (Nelson, 2011). Estradiol has many functions, including aiding in the formation of corpora lutea, affecting the genital tract, affecting several metabolic functions, etc. (Nelson, 2011). These reproductive aspects of hormones are energetically taxing for most species. Thus, it would make sense for gonadal hormones to have an association with body weight and food intake in mammals. In mice, rats, humans, and primates, there are similar associations between sex hormones, food intake, body weight, and adipose tissue. Studies done in one species can be used to further our 
understanding in other mammals that are not yet fully understood, particularly humans. A cross-species comparative analysis is therefore crucial for contributing to our understanding of the physiology of eating.

\section{Effects of Estrogens on Metabolism Across the Menstrual and Estrous Cycle}

Rodents undergo a four-day ovarian and oviduct cycle known as the estrous cycle. Estradiol fluctuates throughout the estrous cycle and can affect food intake in rats based on the stage. During the proestrus stage, characterized by an increase in estradiol, rats tend to consume less food (Butera, 2010). On the other hand, rats tend to increase food intake during the post-ovulatory phase when estradiol levels are low compared to the other peri-ovulatory phase (Brown \& Clegg, 2010; Geary, 2006).

Rats also tend to have maximal wheel running activity during proestrus, while having minimal running activity during the metestrus stage (Anantharaman-Barr \& Decombaz, 1989). This indicates that rats tend to be more active during high levels of estrogens, and other studies have found that ovariectomized female rats exhibit hypoactivity due to the lack of estrogens (Witte et al., 2010). Perhaps activity levels and feeding are affected by estradiol due to unknown potential evolutionary benefits. It would make sense for female mice experiencing higher levels of estradiol and peaking in fertility to focus more energy on mating and less energy on feeding.

The effects of estrous cycle on feeding in mice is similarly confounding. (Butera, 2010) (Witte et al., 2010). While some studies found no difference in food consumption across estrous compared to male mice that do not undergo estrous (Smarr et al., 2019), other studies found an association between estrous levels throughout the estrous cycle and food consumption: there was a decrease in food intake in female mice during the proestrous stage compared to the other stages in the estrous cycle (Butera, 2010; Petersen, 1976; Witte et al., 2010). It is possible that these studies are both correct, but the conditions, such as, but not limited to, the age of the mice, could have been different in each of them and affected the results. These conflicting studies indicate that further studies are needed to determine what the effects of ovarian hormones on food consumption are in female mice.

There also appears to be uncertainty with how hormonal changes during the menstrual cycle affects food consumption. In one study, women appeared to eat less right before ovulation (Roney \& Simmons, 2017). Another study supported this finding and also found that food consumption decreases during the periovulatory stage, and is highest during the luteal stage compared to the other stages (Buffenstein et al., 1995). During the luteal stage, there is a surge of progesterone, and estrogens are not as high in the periovulatory stage. Finally, the premenstrual stage appears to correlate with an increase in food consumption (Klump et al., 2008). This stage corresponds with low levels of progesterone and estrogen. However, other studies have found no correlation between the menstrual cycle and food intake (Souza et al., 2018).

In primates, there appears to be a significant decrease in food intake which is associated with the midcycle estradiol released and the gonadotropin surges that are also experienced (Rosenblatt et al., 1980).

Together, these studies show that tracking food intake across endogenous estrous and menstrual cycles does not yield consistent results, regardless of species. Studies removing and replacing gonadal hormones present a somewhat clearer picture.

\section{The Function of Estrogens and Ovariectomy on Food Intake}

Estrogens influence food intake in some species. In other species, there may not be an effect on food intake, but body weight is affected when estradiol is absent. In rats, ovariectomy (OVX) is shown to increase body weight which is caused by hyperphagia and hypoactivity (Witte et al., 2010). While this weight gain results in an increase in leptin levels for OVX rats compared to sham controls, there is also no change in leptin sensitivity. (Chen \& Heiman, 2001). Leptin is a hormone that is known to contribute to the regulation of body weight (Kratzsch et al., 2000). The change in leptin levels coupled with no change in leptin sensitivity means that the removal of ovarian hormones affect food intake directly. In addition, OVX is also shown to increase meal size (Butera, 2010). These findings link the rat's 
weight gain from OVX to hyperphagia, but there are also findings that demonstrate that food intake eventually returns to baseline levels. One study found that the weight gain occurred during the first five weeks following OVX, but after this time period food intake returned back to normal and "body weight stabilized 12-16\% above sham-control weights for the duration of the experiment” (McElroy \& Wade, 1987). Thus, initially following OVX, hyperphagia and hypoactivity synergize to increase body weight. However, overtime their weight stabilizes, signifying homeostasis has been reached. This might be due to an unknown mechanism that is activated to maintain body weight and feeding in rats that has yet to be studied. Regardless, this signifies that the loss of estradiol and progesterone affects feeding and weight in rats.

In rats, replacement studies demonstrate that estradiol decreases food intake by activation of both $\mathrm{G}$ ProteinCoupled Estrogen Receptor (GPER) and Estrogen Receptor (ER) alpha (Butler et al., 2018). Mice, on the other hand, appear more complicated. The absence of estradiol does not appear to affect food intake in female mice (Witte et al., 2010), though the presence of estradiol can affect food intake and ER alpha is needed for this effect. In fact, when ERalpha is absent, estradiol treatments have no effect on food consumption in female mice while it does have an effect on mice that have ER-alpha (Geary et al., 2001). However, ER-beta, a second nuclear estrogen receptor, does not have to be present for estradiol to affect feeding. In fact, one study was able to determine that ER-alpha had an effect on food intake, but the presence of ER-beta does not affect food intake in ovariectomized rats (Santollo et al., 2007).

The main difference between rats and mice without ovaries is that OVX primarily decreases food intake in rats and instead primarily results in less activity in mice (Witte et al., 2010). While ovariectomy in rats leads to weight gain due to primarily hyperphagia, ovariectomized female mice experience weight gain primarily because of hypoactivity and reduced metabolic rate (Witte et al., 2010). Estradiol itself is known to act on the medial preoptic area and also the ventromedial nucleus of the hypothalamus when the ER-alpha is activated to increase physical activity, so it is understandable that the removal of this hormone through ovariectomy would lead to hypoactivity (Correa et al., 2015; Ogawa et al., 2003). There was no change in food intake despite the absence of ovaries (Witte et al., 2010). The reason why food intake does not change despite the absence of ovaries and compared to the presence of ovaries is still unknown. However, it is possible to learn more about the effects of removing estrogens through other mechanisms such as menopause.

Similar to female rats and mice undergoing ovariectomy, menopause in women is characterized by a decline in reproductive hormones, primarily estradiol, and significant weight gain. Similar to female mice, women entering menopause appear to gain weight despite the fact that food consumption does not change (Davis et al., 2012). When these post-menopausal women are administered estradiol, their metabolic profile of adipose tissue becomes similar to that of pre-menopausal women (see below) (Bjorntorp, 1997). Like mice, women can benefit from a reintroduction of estradiol in the body, which can help with maintaining weight. In fact, it has been stated that female mice are a better representation of women when it comes to the effects of female hormones compared to rats because both mice and humans do not experience a change in food intake but instead a decline in energy expenditure (Massa \& Correa, 2020). In primates, estradiol also appears to show an effect on body weight. In one study, ovariectomized monkeys' body weight decreased with estrogen replacement therapy during the first three months; however, their body weight returned to baseline during the two month recovery period (Sullivan et al., 2012). The percent body fat of these monkeys also appeared to show the same results where body fat levels returned to baseline during the recovery period (Sullivan et al., 2012). With the daily treatment of selective estrogen receptor modulator GSK232802A, the most inactive monkeys and the monkeys who weighed the most seem to have also increased their activity the most (Sullivan et al., 2012). Rhesus monkeys, similar to rats, experience a decrease in food intake and body weight once they undergo estradiol replacement (Czaja \& Goy, 1975). This signifies that the primates are able to maintain or lose weight when administered estrogens.

\section{The Function of Progesterone With and Without the Presence of Estrogens on Food Intake}


Estrogen Therapy is shown to alleviate the effects of ovariectomy on body weight (Hirschberg, 2012), but there are also studies showing how the co-administration of estradiol and progesterone can help with maintaining body weight. As mentioned above, estradiol alone tends to decrease food consumption in some species. In addition, the administration of physiological progesterone alone has no effect on body weight nor food intake in ovariectomized rats subject to time-restricted feeding conditions to induce binge-eating paradigm (Yu et al., 2011). However, when there is cyclic administration of both estradiol and progesterone, these ovariectomized binge-eating rats were able to maintain weight as well as maintain food intake (Yu et al., 2011). This signifies that progesterone does not affect food consumption alone. However, with the presence of estradiol, progesterone does affect food consumption. Furthermore, it is known at this point that estrogens and progesterone work together to affect food intake, but how this happens is still a matter of debate (Yu et al., 2011). One potential reason for how these hormones alter food intake throughout the estrous cycle is potentially "increasing the sensitivity to the satiating signals normally responsible for meal termination" (Drewett, 1974). It has been suggested that estrogens and progesterone are affecting the rate at which food is broken down in the stomach which further affects satiation (Drewett, 1974). Progesterone, generally known to increase body weight with the presence of estradiol, can also help to maintain weight when both estradiol and progesterone are administered to ovariectomized mice.

Progesterone is normally associated with an increase in feeding in humans especially with the presence of estrogens, and estrogens are normally associated with a decrease in feeding (Klump et al., 2008). Most studies done on cycling women were mainly intended to investigate the effects of hormonal contraceptives. However, it is important to note that these contraceptive methods, mainly consisting of administering progesterone, have been shown to lead to weight gain. In cycling women where estrogens are naturally being produced by the body, the hormone progesterone, particularly when it is administered in high doses, tends to increase food intake and leads to weight gain in women (Amatayakul et al., 1980). Similarly, weight gain has mainly been shown when delivering high doses of progesterone to women, such as the contraceptive shot depot medroxyprogesterone acetate (DMPA). Most of the weight gain from synthetic medroxyprogesterone is due to this hormone increasing the amount of fat stored in the body (Amatayakul et al., 1980). In fact, one study found that there was a significant increase in weight among obese women who were administered DMPA compared to the control group (Bonny et al., 2006). In addition, there have been no significant evidence that indicate weight gain in women administered Levonorgestrel, Norethindrone, or DMPA in a separate study (Mainwaring et al., 1995). These conflicting findings may represent an association between the current weight of the individual and how different doses of progesterone might affect women. The study that administered DMPA to obese women also had a separate group of women who were not classified as obese, so this association can be analyzed. Obese women were more sensitive to DMPA and gained more weight compared to non-obese women (Bonny et al., 2006). It would be worth analyzing if overweight individual were also more sensitive to DMPA compared to those with a healthy BMI. However, there has not been a study performed studying this group to the best of our knowledge. As mentioned above, progesterone helped to maintain body weight with the presence of estrogens in female mice. Perhaps a similar approach can be taken in women to determine what dosage is optimal to maintain body weight while concurrently serving as an effective contraceptive.

Although rarely discussed, men also have progesterone and estrogens in their bodies. One of the main functions of progesterone in men is to capacitate sperm (Foresta et al., 1992). Progesterone administration is shown to increase food intake and encourage weight gain in men but further studies are needed (Leinung et al., 1995; Oettel \& Mukhopadhyay, 2010).

Similar to rats, mice, and women, non-human primates also experience progesterone influences on food intake with the presence of estrogens. In some studies, non-human primates have been shown to experience an increase in food intake during the luteal phase, which is characterized by low levels of estradiol but high levels of progesterone compared to the early follicular phase (Rosenblatt et al., 1980). However, one study found that progesterone alone did not affect food intake and progesterone and estrogens administered concurrently did not affect food intake either (Czaja, 1978). It might be worth replicating this experiment because other species have also seen conflicting results across studies. As the function of progesterone seems to alter depending on dosage level and the presence or absence 
of estradiol, replicating these experiments while paying heed to estradiol levels can help strengthen the results of some studies while also refuting the results of other studies regarding the effects of progesterone on food intake. It is important to consider that the methods for both experiments were different, which could be the reason why the results were also different. One of the studies experimentally administered the same daily dose of progesterone and estrogens while the other study took blood samples and observed the natural levels of estrogens and progesterone in the body. The study that administered the daily dose of progesterone did not find any changes in food consumption (Czaja, 1978a). Considering that one of the studies was experimental and the other observational, the effects of progesterone could differ based whether it is naturally or synthetically produced. However, a different approach would need to be done where different levels of progesterone are administered to these non-human primates, since the previous study only administered one constant daily dose of progesterone.

Together, this cross-species analysis supports the idea that progesterone should be considered when looking at the effects of estrogens, since the fluctuating levels of progesterone can also influence food intake.

\section{Function of Testosterone in Food Intake and Body Weight}

The hormone testosterone affects food intake and body weight differently among the sexes and across species. For instance, female rats tend to experience an increase in food intake when testosterone is present along with an increase in body weight (Iwasa et al., 2017). In male rats, the loss of testosterone due to orchiectomy decreases food intake and body weight by decreasing meal frequency which is usually normalized by testosterone (Asarian \& Geary, 2006). Female and male mice are affected by testosterone in a similar manner. Female mice showed an increase in food intake following neonatal exposure to testosterone (Hirschberg, 2012). Another study found similar effects where neonatal female mice who were administered testosterone experienced an increase in food intake 6 weeks after weening (Nohara et al., 2011). Adult female mice experiencing hyperandrogenemia, induced by treatment with the aromatase inhibitor Letrozole, had increased body weight though food intake remained at base level (Skarra et al., 2017). These hyperandrogenemia mice exhibit similar issues, such as insulin resistance related to elevated levels of testosterone in female mammals and can be used as models to further study the effects of polycystic ovarian syndrome in women. Similar to male rats, male mice without testosterone weighed less than male mice with testosterone (Petersen, 1978). In men, there are mixed results on how testosterone affects body weight. Many testosterone treatments have been administered to obese men with a testosterone deficiency. Testosterone deficiency is defined as a lack of function of either or both the testes and/or the hypothalamic pituitary axis, and this condition usually leads to a lack of testosterone levels (Traish \& Zitzmann, 2015). Interestingly, when obese men who lacked testosterone were administered longterm testosterone, they experienced significant weight loss over time (Traish, 2014). The mechanism behind this seemingly bidirectional relationship between testosterone deficiency and obesity is still not very well understood (Traish \& Zitzmann, 2015).

In women, testosterone appears to promote appetite because it increases the frequency of cravings and also increases impulsivity compared to women with typical levels of testosterone (Hirschberg, 2012). One way to observe heightened levels of testosterone in women is by looking at women with polycystic ovary syndrome (PCOS). Women with PCOS also tend to experience hyperinsulinemia which disrupts the GnRH and LH pulse and subsequently leads to hyperandrogenemia (Rojas et al., 2014). The increased levels of testosterone in women experiencing PCOS further aids in insulin resistance creating a positive feedback and subsequently leads to an increase in dietary carbohydrates and lipids consumption, adrenal androgens production, and hormone leptin production (Rojas et al., 2014). Therefore, elevated levels of testosterone in women increases both food intake and weight.

Currently, to our knowledge, studies have not been done that show how testosterone affects food intake in non-human primates. Perhaps performing studies on both female and male non-human primates can help us understand the effects and mechanisms of testosterone on food intake in general.

\section{Other Metabolic Measures: the Interaction of Body Weight, Adipose Tissue, and Hormones}


In rats, both progesterone and estradiol play a role in food intake, body weight, and adiposity. Lipoprotein lipase (LPL) activity is important for adipocyte triglyceride uptake, and it is regulated by both progesterone and estradiol (Schwartz \& Wade, 1981). LPL activity is important to consider since its activity is affected by both progesterone and estradiol and may be one of the reasons why adiposity levels changes when these hormones are present. Ovariectomy increases LPL activity, and estradiol treatment reduces LPL activity (Schwartz \& Wade, 1981). It was also found that neither estrogen treatment alone nor progesterone alone changed parametrial (the fat that surrounds the uterus) adipose tissue LPL activity in weanling rats (Schwartz \& Wade, 1981). Weanling rats overall responded to estrogen treatment the same way as the adult rats; however, LPL activity seem was not affected which could be because of the lesser amounts of hepatic and adipose tissue receptor levels (Schwartz \& Wade, 1981). In addition, there is also a notable increase in both lean and fat mass in rats with estrogen treatment (Chen \& Heiman, 2001). This signifies that estrogens may not contribute to just an increase in adipose tissue but also an increase in other masses unrelated to fat accumulation. These effects are also seen in adipose tissue-specific androgen receptor knockout mice. These mice have a higher intra-adipose estradiol level, which has accordingly been shown to lead to an increase in subcutaneous obesity (Brown \& Clegg, 2010).

In women, estradiol promotes the storage of subcutaneous fat, while the loss of this estradiol during menopause aides with more deposited central fat (Brown \& Clegg, 2010). One reason why women may have a higher amount of subcutaneous fat compared to men is because subcutaneous adipose tissue has a higher concentration of both estrogen receptors and progesterone receptors (Brown \& Clegg, 2010). In addition, women entering menopause are shown to have an increase in visceral fat, which is also seen in ovariectomized rats (Brown \& Clegg, 2010). This occurs because there is a decrease in estradiol in women entering menopause, which means that there will be less subcutaneous adipose tissue. Subcutaneous adipose tissue is also shown to have relatively few androgen receptors, which is another reason why men tend to have less subcutaneous fat (Brown \& Clegg, 2010). In contrast, visceral fat tissue has a higher concentration of androgen receptors and is also more abundant in men (Brown \& Clegg, 2010). In addition, women who exhibit high amounts of testosterone also appear to accumulate visceral fat in the same manner that men do (Rebuffe-Scrive et al, 1989). However, these women also tend to have abnormally low levels of progesterone and estrogens, so this increase in visceral fat in women can also arguably be because of the deficit in estradiol and progesterone (Laatikainen et al., 1980)

Androgen deprivation therapy in men led to weight gain in one particular study with a $2.4-10 \%$ increase of body fat and a 3\% decrease in lean body mass during the 6 month to 2 year duration of this treatment (Kelly \& Jones, 2015). Testosterone production can not only affect body weight, but the inverse relationship can occur. Increased adiposity is directly related to increased leptin levels, and this can result in the hypothalamus becoming leptin resistant (Kelly \& Jones, 2015). This disturbance leads to a decrease in testosterone production (Kelly \& Jones, 2015). Thus, men with high levels of visceral fat tend to have low levels of testosterone compared to men with normal levels of visceral fat (Marin et al., 1995). It has also been observed that obese men with low testosterone levels had higher estradiol levels in their urine compared to the lean control groups (Schneider et al., 1979). However, a more recent study found results that were contrary to this study (Dhindsa et al., 2011). This means that it is still unclear if the aromatization of testosterone (the conversion of testosterone to estradiol) in obese men is occurring at a high rate. Men with a higher Body Mass Index (BMI) tend to experience insulin resistance, which negatively affects Sex Hormone Binding Globulin (SHBG), the protein that binds to sex hormones, and therefore decreases free testosterone levels (Russell \& Grossmann, 2019). However, when testosterone is replaced, there appears to be a significant amount of weight loss along with a decreased waist circumference indicating that the effects caused by the androgen deprivation therapy are reversible (Kelly \& Jones, 2015). Another study administered testosterone to men with low levels of testosterone and found that visceral fat decreased while lean body mass remained the same (Mårin et al., 1993).

\section{Conclusion}


The hormones estrogens, progesterone, and testosterone all affect food intake, body weight, and fat composition across multiple species. The lack of estradiol tends to increase food intake, body weight and fat composition in most species. However, investigations into the effects of estradiol on these parameters conflict. For instance, some studies find that high levels of estrogens decrease food intake in women, while other studies did not find any significant difference in food intake (Roney \& Simmons, 2017). In addition, progesterone does not affect these parameters in all species when it is the only hormone in the body (Yu et al., 2011). However, with the presence of estrogens, progesterone can increase food intake and body weight (Yu et al., 2011). Finally, testosterone generally increases food intake and affects body weight and fat composition differently depending on the sex of the species. In humans, men tend to experience a decrease in fat and fat composition tends to be allocated towards visceral fat (Brown \& Clegg, 2010). Women with high levels of testosterone tend to experience an increase in fat and also experience fat composition allocated towards visceral fat (Brown \& Clegg, 2010).

There have been discrepancies in findings across several studies. For instance, one study found that women administered the progestin DMPA experienced a significant increase in weight (Bonny et al., 2006) while a separate study found no significant difference (Mainwaring et al., 1995). Another discrepancy can be seen among men and women when examining the effects of testosterone on body composition and food consumption. Men with higher levels of testosterone were correlated to be at healthier BMI levels compared to their counterparts who have low levels of testosterone (Traish \& Zitzmann, 2015). In contrast, women with PCOS, which includes abnormally high levels of testosterone in the body, can experience weight gain (Rojas et al., 2014). A discrepancy in the findings of one study can be cross analyzed with a similar study in a different species, and the knowledge gap can be bridged in that matter. For example, our results regarding the effects of DMPA among cycling women can be cross-analyzed with non-human cycling primates who were administered progesterone. Non-human cycling primates did not experience an effect in body weight when administered progesterone, which is in accordance to the study that found no significant difference in women administered DMPA (Czaja, 1978). When there are conflicting findings within a species group, a crossspecies analysis gives one the ability to decide which finding is stronger and supported across multiple species. Discrepancies among the sexes are also important to consider because there can potentially be a mechanism in the body that leads to a higher risk of being overweight in one sex while helping maintain weight in the other sex.

While there are plenty of studies that have been performed on humans, there are limitations on what can be studied. For example, a lot of human studies mentioned had a primary purpose, such as studying the effectiveness of contraceptives (Amatayakul et al., 1980) or alleviating the negative effects of low testosterone in men such as unwanted weight gain (Traish \& Zitzmann, 2015). None of the studies mentioned above had a primary focus on the relation on food intake and hormones because these types of studies are viewed as subsidiary issues such as side effects of a medication or condition such as PCOS (Rojas et al., 2014). Relegating these studies to secondary measures limits our full understanding because of their correlative nature. This means that the results needed to better understand the physiology of eating cannot be found without performing an actual experiment.

While there are numerous studies relating hormones to food intake and body weight, it seems that there is still some uncertainty on how female hormones affect mice and humans. Some studies found an effect while others did not. It is possible that a compensatory mechanism in the body is preventing these female hormones from affecting food consumption and body weight when no effect is found (De Vries, 2004). This mechanism has yet to be found, but it may explain the inconsistencies found in several studies performing similar experiments. For example, some studies state that women do not experience a change in food intake across the menstrual cycle despite fluctuations in female hormones (Souza et al., 2018). However, based on the same experiment performed on other species, it is apparent that the same hormone produced similar effects in the other species where the results were consistent. These consistent results among several species can bridge the gap that was not found in the inconsistent results in other species. However, it is important to consider that these inconsistences may have occurred because of the differences in experimental approach. When replicating these experiments, it is important to consider an alternate approach to further contribute to our understanding of these past findings. However, knowing the variety of approaches and results 
for the topic being studied can help with determining what needs to be changed from the methods of these studies to yield consistent results. For this reason, a cross-species analysis is deemed useful and insightful.

\section{References}

Amatayakul, K., Sivasomboon, B., \& Thanangkul, O. (1980). A study of the mechanism of weight gain in medroxyprogesterone acetate users. Contraception, 22(6), 605-622. https://doi.org/10.1016/00107824(80)90087-6

Anantharaman-Barr, H. G., \& Decombaz, J. (1989). The effect of wheel running and the estrous cycle on energy expenditure in female rats. Physiology and Behavior, 46(2), 259-263. https://doi.org/10.1016/00319384(89)90265-5

Asarian, L., \& Geary, N. (2006). Modulation of appetite by gonadal steroid hormones. Philosophical Transactions of the Royal Society B: Biological Sciences, 361(1471), 1251-1263. https://doi.org/10.1098/rstb.2006.1860

Bjorntorp, P. (n.d.). Hormonal control of regional fat distribution. Retrieved July 26, 2020, from https://academic.oup.com/humrep/article-abstract/12/suppl_1/21/693260

Bonny, A. E., Ziegler, J., Harvey, R., Debanne, S. M., Secic, M., \& Cromer, B. A. (2006). Weight Gain in Obese and Nonobese Adolescent Girls Initiating Depot Medroxyprogesterone, Oral Contraceptive Pills, or No Hormonal Contraceptive Method A. In Arch Pediatr Adolesc Med (Vol. 160).

Brown, L. M., \& Clegg, D. J. (2010). Central effects of estradiol in the regulation of food intake, body weight, and adiposity. In Journal of Steroid Biochemistry and Molecular Biology (Vol. 122, Issues 1-3, pp. 65-73). https://doi.org/10.1016/j.jsbmb.2009.12.005

Buffenstein, R., Poppitt, S. D., McDevitt, R. M., \& Prentice, A. M. (1995). Food intake and the menstrual cycle: A retrospective analysis, with implications for appetite research. Physiology and Behavior, 58(6), $1067-1077$. https://doi.org/10.1016/0031-9384(95)02003-9

Butera, P. C. (2010). Estradiol and the control of food intake. Physiology and Behavior, 99(2), 175-180. https://doi.org/10.1016/j.physbeh.2009.06.010

Butler, M. J., Hildebrandt, R. P., \& Eckel, L. A. (2018). Selective activation of estrogen receptors, ER $\alpha$ and GPER1, rapidly decreases food intake in female rats. Hormones and Behavior, 103, 54-61. https://doi.org/10.1016/j.yhbeh.2018.05.018

Chen, Y., \& Heiman, M. L. (2001). Increased weight gain after ovariectomy is not a consequence of leptin resistance. American Journal of Physiology - Endocrinology and Metabolism, 280(2 43-2). https://doi.org/10.1152/ajpendo.2001.280.2.e315

Correa, S. M., Newstrom, D. W., Warne, J. P., Flandin, P., Cheung, C. C., Lin-Moore, A. T., Pierce, A. A., Xu, A. W., Rubenstein, J. L., \& Ingraham, H. A. (2015). An estrogen-responsive module in the ventromedial hypothalamus selectively drives sex-specific activity in females. Cell Reports, 10(1), 62-74. https://doi.org/10.1016/j.celrep.2014.12.011

Czaja, J. A. (1978). Ovarian influences on primate food intake: Assessment of progesterone actions. Physiology and Behavior, 21(6), 923-928. https://doi.org/10.1016/0031-9384(78)90167-1

Czaja, J. A., \& Goy, R. W. (1975). Ovarian hormones and food intake in female guinea pigs and rhesus monkeys. Hormones and Behavior, 6(4), 329-349. https://doi.org/10.1016/0018-506X(75)90003-3

Davis, S. R., Castelo-Branco, C., Chedraui, P., Lumsden, M. A., Nappi, R. E., Shah, D., \& Villaseca, P. (2012). Understanding weight gain at menopause. https://doi.org/10.3109/13697137.2012.707385

De Vries, G. J. (2004). Minireview: Sex Differences in Adult and Developing Brains: Compensation, Compensation, Compensation. In Endocrinology (Vol. 145, Issue 3, pp. 1063-1068). Oxford Academic. https://doi.org/10.1210/en.2003-1504

Dhindsa, S., Furlanetto, R., Vora, M., Ghanim, H., Chaudhuri, A., \& Dandona, P. (2011). Low estradiol concentrations in men with subnormal testosterone concentrations and type 2 diabetes. Diabetes Care, 34(8), 1854-1859. https://doi.org/10.2337/dc11-0208

Drewett, R. F. (1974). The meal patterns of the oestrous cycle and their motivational significance. QUART.J.EXP.PSYCHOL., 26(3), 489-494. https://doi.org/10.1080/14640747408400438

Foresta, C., Rossato, M., Mioni, R., \& Zorzi, M. (1992). Progesterone induces capacitation in human spermatozoa. Andrologia, 24(1), 33-35. https://doi.org/10.1111/j.1439-0272.1992.tb02605.x

Geary, N. (2006). The Estrogenic Inhibition of Eating. In Neurobiology of Food and Fluid Intake (pp. 307-345). Kluwer Academic Publishers. https://doi.org/10.1007/0-306-48643-1_12

Geary, N., Asarian, L., Korach, K. S., Pfaff, D. W., \& Ogawa, S. (2001). Deficits in E2-dependent control of 
feeding, weight gain, and cholecystokinin satiation in ER- $\alpha$ null mice. In Endocrinology (Vol. 142, Issue 11). https://doi.org/10.1210/endo.142.11.8504

Hirschberg, A. L. (2012). Sex hormones, appetite and eating behaviour in women. In Maturitas (Vol. 71, Issue 3, pp. 248-256). Elsevier. https://doi.org/10.1016/j.maturitas.2011.12.016

Iwasa, T., Matsuzaki, T., Yiliyasi, M., Yano, K., \& Irahara, M. (2017). The effects of chronic testosterone administration on body weight, food intake, and fat weight were age-dependent. Steroids. https://doi.org/10.1016/j.steroids.2017.08.014

Kelly, D. M., \& Jones, T. H. (2015). Testosterone and obesity. Obesity Reviews, 16(7), 581-606. https://doi.org/10.1111/obr.12282

Klump, K. L., Keel, P. K., Culbert, K. M., \& Edler, C. (2008). Ovarian hormones and binge eating: Exploring associations in community samples. Psychological Medicine, 38(12), 1749-1757. https://doi.org/10.1017/S0033291708002997

Kratzsch, J., Höckel, M., \& Kiess, W. (2000). Leptin and pregnancy outcome. In Current Opinion in Obstetrics and Gynecology (Vol. 12, Issue 6, pp. 501-505). Curr Opin Obstet Gynecol. https://doi.org/10.1097/00001703200012000-00008

Laatikainen, T. J., Apter, D. L., Paavonem, J. A., \& Wahlstrom, T. R. (1980). Steroids in Ovarian and Peripheral Venous Blood in Polycystic Ovarian Disease. Clinical Endocrinology, 13(2), 125-134. https://doi.org/10.1111/j.1365-2265.1980.tb01033.x

Leinung, M. C., Liporace, R., \& Miller, C. H. (1995). Induction of adrenal suppression by megestrol acetate in patients with AIDS. Annals of Internal Medicine, 122(11), 843-845. https://doi.org/10.7326/0003-4819-12211-199506010-00006

Mainwaring, R., Hales, H. A., Stevenson, K., Hatasaka, H. H., Poulson, A. M., Jones, K. P., \& Peterson, C. M. (1995). Metabolic parameter, bleeding, and weight changes in U.S. women using progestin only contraceptives. Contraception, 51(3), 149-153. https://doi.org/10.1016/0010-7824(95)00011-X

Mårin, P., Holmtäng, S., Gustafsson, C., Jönsson, L., Kvist, H., Elander, A., Eldh, J., Sjöström, L., Holm, G., \& Björntorp, P. (1993). Androgen Treatment of Abdominally Obese Men. Obesity Research, 1(4), 245-251. https://doi.org/10.1002/j.1550-8528.1993.tb00618.x

Marin, P., Oden, B., \& Bjorntorp, P. (1995). Assimilation and mobilization of triglycerides in subcutaneous abdominal and femoral adipose tissue in vivo in men: Effects of androgens. In Journal of Clinical Endocrinology and Metabolism (Vol. 80, Issue 1). https://doi.org/10.1210/jcem.80.1.7829619

Massa, M. G., \& Correa, S. M. (2020). Sexes on the brain: Sex as multiple biological variables in the neuronal control of feeding. In Biochimica et Biophysica Acta - Molecular Basis of Disease (Vol. 1866, Issue 10). Elsevier B.V. https://doi.org/10.1016/j.bbadis.2020.165840

McElroy, J. F., \& Wade, G. N. (1987). Short- and long-term effects of ovariectomy on food intake, body weight, carcass composition, and brown adipose tissue in rats. Physiology and Behavior, 39(3), 361-365. https://doi.org/10.1016/0031-9384(87)90235-6

Nelson, R. J. (2011). An Introduction to Behavioral Endocrinology. 4.

Nohara, K., Zhang, Y., Waraich, R. S., Laque, A., Tiano, J. P., Tong, J., Münzberg, H., \& Mauvais-Jarvis, F. (2011). Early-Life Exposure to Testosterone Programs the Hypothalamic Melanocortin System. Endocrinology, 152(4), 1661-1669. https://doi.org/10.1210/en.2010-1288

Oettel, M., \& Mukhopadhyay, A. K. (2010). The Aging Male Progesterone: the forgotten hormone in men? Progesterone: the forgotten hormone in men? https://doi.org/10.1080/13685530400004199

Ogawa, S., Chan, J., Gustafsson, J. Å., Korach, K. S., \& Pfaff, D. W. (2003). Estrogen increases locomotor activity in mice through estrogen receptor $\alpha$ : Specificity for the type of activity. Endocrinology, 144(1), 230-239. https://doi.org/10.1210/en.2002-220519

Petersen, S. (1976). The temporal pattern of feeding over the oestrous cycle of the mouse. Animal Behaviour, 24(4), 939-955. https://doi.org/10.1016/S0003-3472(76)80023-1

Petersen, S. (1978). Effects of testosterone upon feeding in male mice. Animal Behaviour, 26(PART 3), 945-952. https://doi.org/10.1016/0003-3472(78)90158-6

Rojas, J., Chávez, M., Olivar, L., Rojas, M., Morillo, J., Mejías, J., Calvo, M., \& Bermúdez, V. (2014). Polycystic Ovary Syndrome, Insulin Resistance, and Obesity: Navigating the Pathophysiologic Labyrinth. International Journal of Reproductive Medicine, 2014, 1-17. https://doi.org/10.1155/2014/719050

Roney, J. R., \& Simmons, Z. L. (2017). Ovarian hormone fluctuations predict within-cycle shifts in women's food intake. Hormones and Behavior, 90, 8-14. https://doi.org/10.1016/j.yhbeh.2017.01.009

Rosenblatt, H., Dyrenfurth, I., Ferin, M., \& Vande Wiele, R. L. (1980). Food intake and the menstrual cycle in rhesus monkeys. Physiology and Behavior, 24(3), 447-449. https://doi.org/10.1016/0031-9384(80)90234-6 


\section{Journal of Student Research}

Russell, N., \& Grossmann, M. (2019). Estradiol as a male hormone. In European Journal of Endocrinology (Vol. 181, Issue 1, pp. R23-R43). BioScientifica Ltd. https://doi.org/10.1530/EJE-18-1000

Santollo, J., Wiley, M. D., \& Eckel, L. A. (2007). Acute activation of ER $\alpha$ decreases food intake, meal size, and body weight in ovariectomized rats. American Journal of Physiology - Regulatory Integrative and Comparative Physiology, 293(6). https://doi.org/10.1152/ajpregu.00385.2007

Schneider, G., Kirschner, M. A., Berkowitz, R., \& Ertel, N. H. (1979). Increased estrogen production in obese men. Journal of Clinical Endocrinology and Metabolism, 48(4), 633-638. https://doi.org/10.1210/jcem-48-4-633

Schwartz, S. M., \& Wade, G. N. (1981). Effects of estradiol and progesterone on food intake, body weight, and carcass adiposity in weanling rats. American Journal of Physiology - Endocrinology and Metabolism, 3(5). https://doi.org/10.1152/ajpendo.1981.240.5.e499

Skarra, D. V., Hernández-Carretero, A., Rivera, A. J., Anvar, A. R., \& Thackray, V. G. (2017). Hyperandrogenemia induced by letrozole treatment of pubertal female mice results in hyperinsulinemia prior to weight gain and insulin resistance. Endocrinology, 158(9), 2988-3003. https://doi.org/10.1210/en.2016-1898

Smarr, B., Rowland, N. E., \& Zucker, I. (2019). Male and female mice show equal variability in food intake across 4-day spans that encompass estrous cycles. PLOS ONE, 14(7), e0218935. https://doi.org/10.1371/journal.pone.0218935

Souza, L. B. De, Martins, K. A., Cordeiro, M. M., Rodrigues, Y. D. S., Rafacho, B. P. M., \& Bomfim, R. A. (2018). Do Food Intake and Food Cravings Change during the Menstrual Cycle of Young Women? Revista Brasileira de Ginecologia e Obstetricia, 40(11), 686-692. https://doi.org/10.1055/s-0038-1675831

Sullivan, E. L., Shearin, J., Koegler, F. H., \& Cameron, J. L. (2012). Selective estrogen receptor modulator promotes weight loss in ovariectomized female rhesus monkeys (Macaca mulatta) by decreasing food intake and increasing activity. American Journal of Physiology-Endocrinology and Metabolism, 302(7), E759-E767. https://doi.org/10.1152/ajpendo.00327.2011

Traish, A. M. (2014). Testosterone and weight loss. Current Opinion in Endocrinology \& Diabetes and Obesity, 21(5), 313-322. https://doi.org/10.1097/MED.0000000000000086

Traish, A. M., \& Zitzmann, M. (2015). The complex and multifactorial relationship between testosterone deficiency (TD), obesity and vascular disease. In Reviews in Endocrine and Metabolic Disorders (Vol. 16, Issue 3, pp. 249-268). Springer New York LLC. https://doi.org/10.1007/s11154-015-9323-2

Witte, M. M., Resuehr, D., Chandler, A. R., Mehle, A. K., \& Overton, J. M. (2010). Female mice and rats exhibit species-specific metabolic and behavioral responses to ovariectomy. General and Comparative Endocrinology. https://doi.org/10.1016/j.ygcen.2010.01.006

Yu, Z., Geary, N., \& Corwin, R. L. (2011). Individual effects of estradiol and progesterone on food intake and body weight in ovariectomized binge rats. Physiology and Behavior, 104(5), 687-693. https://doi.org/10.1016/j.physbeh.2011.07.017 